\title{
ON TRULY NONLINEAR OSCILLATOR EQUATIONS OF ERMAKOV-PINNEY TYPE
}

\section{MARCELLIN NONTI, KOLAWOLE KEGNIDE DAMIEN ADJAÏ, JEAN AKANDE, MARC DELPHIN MONSIA*}

\author{
Department of Physics, University of Abomey-Calavi, Abomey-Calavi, 01.BP.526, Cotonou, Benin
}

${ }^{*}$ Corresponding author: monsiadelphin@yahoo.fr

\begin{abstract}
In this paper we present a general class of differential equations of Ermakov-Pinney type which may serve as truly nonlinear oscillators. We show the existence of periodic solutions by exact integration after the phase plane analysis. The related quadratic Lienard type equations are examined to show for the first time that the Jacobi elliptic functions may be solution of second-order autonomous non-polynomial differential equations.
\end{abstract}

\section{INTRODUCTION}

In the research field of periodic solution to Lienard nonlinear differential equations of the form

$$
\ddot{x}+f(x)=0
$$

where the overdot stands for the derivative with respect to time, and $f(x)$ is a nonlinear function of $x$, it is less usual to notice differential equations with exact periodic solutions. It is again very less usual to find differential equations with exact periodic solutions in terms of trigonometric functions. This makes the Ermakov-Pinney equation

$$
\ddot{x}+a x+\frac{b}{x^{3}}=0
$$

Received October $5^{\text {th }}, 2021$; accepted November $2^{\text {nd }}, 2021$; published November $19^{\text {th }}, 2021$.

2010 Mathematics Subject Classification. 34C15, 34C25.

Key words and phrases. Lienard equations; Ermakov-Pinney equation; truly nonlinear oscillators; periodic solution.

(C)2021 Authors retain the copyrights of their papers, and all open access articles are distributed under the terms of the Creative Commons Attribution License. 
unusual and underlines its high usefulness in science and engineering. In this way a lot of applications in classical mechanics as well as in quantum mechanics for example, has been carried out during these decades ( [1], [2]). The Ermakov-Pinney equation (1.2) has been studied in [2] to show the existence of new periodic solutions and non-periodic solutions. In [3] an exceptional Lienard equation with strong and high order nonlinearity is presented. A Lienard equation with a periodic solution in terms of a single trigonometric function, which may lead to a quadratic Lienard type equation with a periodic solution exhibiting harmonic oscillations, and contains several well-known equations like the Ermakov-Pinney equation [4], the Mickens truly nonlinear oscillators and the cubic Duffing equation as special cases, has never been highlighted in the literature despite the well established theory of differential equations, as it is carried out in [3]. According to $([5],[6])$ all Ermakov-Pinney equations may be reduced to

$$
\ddot{x}+\frac{b}{x^{3}}=0
$$

using a variable change. One may say that the cubic singularity defines the nonlinear property of the Ermakov-Pinney equation. So a differential equation with cubic nonlinearity may be said of Ermakov-Pinney type. In this perspective consider the Lienard differential equation ( [2], [3])

$$
\ddot{x}+\frac{1}{2}(\alpha-q) a x^{\alpha-q-1}+\frac{q b}{2} x^{-q-1}=0
$$

Making $q=2$, yields as equation

$$
\ddot{x}+\frac{1}{2}(\alpha-2) a x^{\alpha-3}+\frac{b}{x^{3}}=0
$$

In view of the above, the equation (1.5) may be characterized of Ermakov-Pinney type. For $\alpha=2$, the equation (1.5) reduces to (1.3). The equation (1.5) may be reduced to a quadrature. Using the corresponding first integral ( [2], [3])

$$
\dot{x}^{2} x^{2}+a x^{\alpha}=b
$$

the general solution of (1.5) is written as the quadrature defined by

$$
\pm(t+K)=\int \frac{x d x}{\sqrt{b-a x^{\alpha}}}
$$

Let

$$
J=\int \frac{x d x}{\sqrt{b-a x^{\alpha}}}
$$


The change of variable $X^{2}=b-a x^{\alpha}$, and $d x=-\frac{2}{a \alpha}\left(\frac{b-X^{2}}{a}\right)^{\frac{1-\alpha}{\alpha}} X d X$, where $a \neq 0$, and $\alpha \neq 0$, turns $J$ into

$$
J=-\frac{2}{\alpha a^{\frac{2}{\alpha}}} \int\left(b-X^{2}\right)^{\frac{2-\alpha}{\alpha}} d X
$$

A new change of variable $\phi=\frac{X}{\sqrt{b}}$, where $b \neq 0$, reduces $J$ to the form

$$
J=-\frac{2 \sqrt{b}}{b \alpha}\left(\frac{b}{a}\right)^{\frac{2}{\alpha}} \int\left(1-\phi^{2}\right)^{\frac{2-\alpha}{\alpha}} d \phi
$$

As can be seen, the value of the integral in $J$ could not be known exactly. A new change of variable in terms of trigonometric or hyperbolic functions may be also performed but this does not solve the problem. However, it shows that the general solutions of some specific equations of the equation (1.5) are not periodic. The equation (1.5) may be reduced to the form

$$
\ddot{x}+(n-1) a x^{2 n-3}+\frac{b}{x^{3}}=0
$$

where $\alpha=2 n, n$ is an integer. The equation (1.11) may be of physical importance since it has the structure of truly nonlinear oscillators formulated by Mickens in his book [7], and contains the famous ErmakovPinney equation as special case. It is known also that differential equations with power nonlinearities are often encountered in mathematical modeling of physical problems. A vast literature exists on the topic of truly nonlinear oscillators. During the last decades many authors investigated these nonlinear differential equations. As nonlinear differential equations, they have no exact explicit solutions in general. Moreover they could not be solved by the well known standard approximate analytical techniques [7]. So the existence of periodic solutions of these equations is yet under some debate. This particularly, becomes an attractive research problem when the second order autonomous truly nonlinear equation has a singularity at the origin and can have no critical point, necessary condition, according to [8], for a planar autonomous systems to have a periodic solution. It was the case of the so-called pseudo-oscillator investigated in [8]. The authors [8] concluded that such a differential equation has no periodic solution. In contrast to this the author in [9] showed that periodic solution exists, at least a non-smooth solution. The author [9] carried out a theory to build such periodic solutions. In [10] the two general solutions predicted in [8] have been exactly calculated and the authors [10] concluded also to the non existence of smoth periodic solution. The equation (1.11) has a cubic singularity at the origin for a positive integer $n$, but may have fixed points. For $n$ negative, singularities appear also. Choosing $n=1$, that is $\alpha=2$, reduces the equation (1.11) to the Ermakov-Pinney 
type equation (1.3). For $n=2$, that is $\alpha=4$, the equation (1.11) becomes the Ermakov-Pinney equation (1.2). Using $n=3$, in other words $\alpha=6$, the equation (1.11) transforms into

$$
\ddot{x}+2 a x^{3}+\frac{b}{x^{3}}=0
$$

For $b=0$, the equation (1.12) reduces to the well-known restricted cubic Duffing equation ( [7], [11], [12]) for which it is said that all the solutions are periodic. Contrary to these authors and to several others, it has been shown that such an equation may exhibit non-periodic solution, precisely complex-valued solutions [13]. Putting $n=4$, into the equation (1.11), yields

$$
\ddot{x}+3 a x^{5}+\frac{b}{x^{3}}=0
$$

When $b=0$, the equation (1.13) reduces to the restricted quintic Duffing equation. The equations (1.12), (1.13) and others, which may be obtained for various values of $n$, have not been previously studied in the literature. The above underlines the mathematical importance to ask whether the equation (1.11) has periodic solutions for $n \succ 2$. The case $n=2$, corresponding to the Ermakov-Pinney equation has been investigated in several papers. Periodic solutions have been carried out for this equation in ( [2], [4]). The case of negative $n$ will be considered separately in a paper. In the present work we show that the equation (1.11) may exhibit periodic and explicit general solutions for $\frac{a}{b}=1$, and $2 \preceq n \preceq 4$. The choice of negative $b$ has no physical sense as we can see below. In this regard the qualitative properties of solutions of the equation (1.11) are investigated in section (2), and the section (3) is devoted to exhibit exact periodic solutions of (1.11) under the preceding conditions. The general solutions of the equation (1.3) are also exhibited in this section. Finally a conclusion is formulated for the work.

\section{Qualitative Properties of SOlutions}

The qualitative properties of solutions to (1.11) are investigated in this section using the phase plane method. Therefore the equation (1.11) is equivalent to the planar autonomous dynamical system

$$
\left\{\begin{array}{l}
\dot{x}=y \\
\dot{y}=-(n-1) a x^{2 n-3}-\frac{b}{x^{3}}
\end{array}\right.
$$

The fixed point is defined by $y=0$ and $x=\left[-\frac{b}{(n-1) a}\right]^{\frac{1}{2 n}}$. As one may see, for $\frac{b}{a}=-1$, the critical point is real, but for $\frac{b}{a}=1$, the coordinate $x$ may become complex. From (2.1) one may write 


$$
\frac{d y}{d x}=-\frac{(n-1) a x^{2 n}+b}{y x^{3}}
$$

The separation of variable leads to

$$
y d y=-\left[(n-1) a x^{2 n-3}+\frac{b}{x^{3}}\right] d x
$$

By integration, one may obtain the integral curves given by

$$
y^{2}=-a x^{2 n-2}+\frac{b}{x^{2}}+c
$$

This means, according to the equation (1.6) that the integration constant can be choosen as $c=0$, so that the Hamiltonian of the system can be written

$$
H=\dot{x}^{2} x^{2}+a x^{2 n}
$$

The Hamiltonian (2.4) is time independent such that the equation (1.11) defines a conservative system from physical point of view. In this context, one can expect the existence of periodic solutions to the equation (1.11). Now the objective is to calculate the exact and explicit general solutions of the equations (1.3), (1.12) and (1.13).

\section{General solutions}

3.1. General solution of (1.3). The reduced Ermakov-Pinney equation (1.3) has been the object of a high consideration in the literature since the Ermakov-Pinney equations can be reduced to (1.3) as mentioned before. In order to study its properties, the Ermakov-Pinney equation (1.3) for $b=-1$, in ( [5], [6]) is transformed into

$$
2 z \ddot{z}-3 \dot{z}^{2}+4 z^{4}=0
$$

using the point transformation

$$
z=\frac{1}{x^{2}}
$$

However, no explicit general solution is given. In [6] the equation (3.1) is examined from symmetry group point of view and the authors [6] arrived to examine

$$
2 z \ddot{z}-3 \dot{z}^{2}+B z^{4}=0
$$


where $B$ is the arbitrary constant. The authors [6] observe that the analysis of Lie point symmetries is not adequate for (3.3) which requires, rather than nonlocal symmetry calculation. As the evaluation of nonlocal symmetries may be complicated, the authors [6] apply the Jacobi last multiplier approach to find the solution of (3.1) in terms of time dependent integral. On the other hand, the equation (1.3) is also investigated in [14]. The authors [14] succeed to calculate a general solution of (1.3) where $b=1$, in terms of the so-called $C$ invariant related to the Ermakov-Pinney invariant. However, the point transformation of the equation (1.3) into (3.1) shows clearly that such an equation has two general solutions which differ only by a sign. The objective in this paragraph is to determine in a direct fashion the two general solutions and by exactly solving the auxiliary equation (3.1), in terms of arbitrary constants.

3.1.1. Direct method. According to (1.10) the equation (1.3) may be reduced to the quadrature

$$
\pm(t+K)=-\frac{\sqrt{b}}{b}\left(\frac{b}{a}\right) \int d \phi
$$

which leads to

$$
-\frac{\sqrt{b}}{b}\left(\frac{b}{a}\right) \phi= \pm(t+K)
$$

Using the change $\phi=\frac{X}{\sqrt{b}}=\frac{X \sqrt{b}}{b}$, the equation (3.5) gives

$$
X= \pm a(t+K)
$$

from which, using the previous relation $X^{2}=b-a x^{2}$, one may secure the general solutions of (1.3) as

$$
x(t)= \pm\left[\frac{b}{a}-a(t+K)^{2}\right]^{\frac{1}{2}}
$$

where $K$ is an integration constant.

3.1.2. Solution using the auxiliary equation (3.1). Let us consider the generalized Sundman transformation theory introduced recently in the literature by Akande and coworkers [15]. In fact the generalized Sundman transformation is a powerfull change of variables which allows solving differential equations with a few mathematical manipulations. In the theory introduced by Akande et al. [15] the oscillator harmonic equation

$$
\ddot{u}+\omega^{2} u=0
$$

where $\omega$ is a constant, $\ddot{u}$ means the second derivative with respect to $\tau$, and

$$
u(\tau)=A_{0} \sin (\omega \tau+\beta)
$$

is transformed, under the change of variables 


$$
u(\tau)=F(t, z), \quad d \tau=G(t, z) d t
$$

where

$$
F(t, z)=\int g(z)^{l} d z, \quad G(t, z)=e^{\gamma \varphi(z)}, \quad \text { and } \quad \frac{\partial F(t, z)}{\partial z} \neq 0
$$

to the second order differential equation

$$
\ddot{z}+\left(l \frac{g^{\prime}(z)}{g(z)}-\gamma \varphi^{\prime}(z)\right) \dot{z}^{2}+\omega^{2} \frac{\exp (2 \gamma \varphi(z)) \int g(z)^{l} d z}{g(z)^{l}}=0
$$

where $A_{0}, \beta, l, \gamma$ are arbitrary parameters, $g(z) \neq 0$, and $\varphi(z)$ are arbitrary functions of $z$, and prime denotes differentiation with respect to $z$. The application of $\varphi(z)=\ln (f(z))$, leads to

$$
\ddot{z}+\left(l \frac{g^{\prime}(z)}{g(z)}-\gamma \frac{f^{\prime}(z)}{f(z)}\right) \dot{z}^{2}+\omega^{2} \frac{f(z)^{2 \gamma} \int g(z)^{l} d z}{g(z)^{l}}=0
$$

Putting $g(z)=z$, and $f(z)=z^{2}$, into (3.13), allows one to obtain

$$
\ddot{z}+(l-2 \gamma) \frac{\dot{z}^{2}}{z}+\frac{\omega^{2}}{l+1} z^{4 \gamma+1}=0
$$

which may reduce, choosing $\gamma=-l=\frac{1}{2}$, to

$$
\ddot{z}-\frac{3}{2} \frac{\dot{z}^{2}}{z}+2 \omega^{2} z^{3}=0
$$

The equation (3.15) may be identified to (3.3) when $4 \omega^{2}=B$ and to (3.1) when $\omega^{2}=1$. So from the solutions of (3.15) one may deduce those of (1.3) with $b=-1$. In this context the transformation defined by (3.10) and (3.11) becomes

$$
u(\tau)=\int z^{-\frac{1}{2}} d z=2 z^{\frac{1}{2}}
$$

that is, $z=\frac{u^{2}}{4}$, and $d \tau=z d t$, so that the equation (3.15) may be reduced to the quadrature determined by

$$
\frac{A_{0}^{2}}{4}\left(t+K_{1}\right)=\int \frac{d \tau}{\sin ^{2}(\omega \tau+\beta)}
$$

where $K_{1}$ is an integration constant. The change of variable $s=\omega \tau+\beta$, reduces the integral in (3.16) to 


$$
J=\frac{1}{\omega} \int \frac{d s}{\sin ^{2}(s)}
$$

which yields

$$
-\cot (s)=\frac{\omega A_{0}^{2}}{4}\left(t+K_{1}\right)
$$

such that

$$
\omega \tau+\beta=\cot ^{-1}\left[-\frac{\omega A_{0}^{2}}{4}\left(t+K_{1}\right)\right]
$$

Substituting (3.19) into (3.9) yields the general solution to (3.15) as

$$
z(t)=\frac{1}{4} A_{0}^{2} \sin ^{2}\left[\cot ^{-1}\left(-\frac{\omega A_{0}^{2}}{4}\left(t+K_{1}\right)\right)\right]
$$

Using (3.20) one may deduce the solution of (3.1) in the form

$$
z(t)=\frac{1}{4} A_{0}^{2} \sin ^{2}\left[\cot ^{-1}\left(-\frac{A_{0}^{2}}{4}\left(t+K_{1}\right)\right)\right]
$$

Therefore the solution of (1.3) where $b=-1$, becomes

$$
x(t)^{2}=\frac{4}{A_{0}^{2} \sin ^{2}\left[\cot ^{-1}\left(-\frac{A_{0}^{2}}{4}\left(t+K_{1}\right)\right)\right]}
$$

that is

$$
x(t)= \pm \frac{2}{A_{0} \sin \left[\cot ^{-1}\left(-\frac{A_{0}^{2}}{4}\left(t+K_{1}\right)\right)\right]}
$$

Knowing $-\cot ^{-1}(\nu)=\sin ^{-1}\left(\frac{1}{\sqrt{1+\nu^{2}}}\right)$, for $\nu \succ 0$, and $\cot ^{-1}(\nu)=\pi-\sin ^{-1}\left(\frac{1}{\sqrt{1+\nu^{2}}}\right)$, for $\nu \prec 0$, then the general solutions (3.23) reduce to

$$
x(t)= \pm \frac{2 \sqrt{1+\frac{A_{0}^{4}}{16}\left(t+K_{1}\right)^{2}}}{A_{0}}
$$

Now the problem to be solved is to calculate the general solution of the equations (1.12) and (1.13) to show analytically and explicitly the existence of periodic solutions to the equation (1.11). 


\subsection{Exact periodic and complex-valued solutions.}

3.2.1. Periodic and complex-valued solutions of (1.12). The equation (1.12) is obtained when $n=3$, that is when $\alpha=6$, from the equation (1.11). Two cases may be investigated.

\section{Periodic solution}

For reason of simplicity we choose $a=b=1$. In this case the integral $J$ becomes

$$
J=-\frac{1}{3} \int \frac{d \phi}{\sqrt[3]{\left(1-\phi^{2}\right)^{2}}}
$$

which may be rewritten as [17]

$$
J=-\frac{1}{3} \int_{0}^{\phi} \frac{d \xi}{\sqrt[3]{\left(1-\xi^{2}\right)^{2}}}= \pm\left(t+c_{1}\right)
$$

where $0 \prec \phi \prec \infty$, and $c_{1}$ is an arbitrary parameter.

By integration, (3.26) reduces to [17]

$$
J=\frac{1}{3} \frac{3}{2 \sqrt[4]{3}} F(\psi, k)
$$

where $\psi=\cos ^{-1}\left[\frac{\sqrt{3}-1-\sqrt[3]{\phi^{2}-1}}{\sqrt{3}+1+\sqrt[3]{\phi^{2}-1}}\right]$, and $k^{2}=\frac{2+\sqrt{3}}{4}$. Using (3.27), one may write

$$
J=\frac{1}{2 \sqrt[4]{3}} F(\psi, k)= \pm\left(t+c_{1}\right)
$$

from which one may get

$$
\cos \psi=\operatorname{cn}\left[ \pm 2 \sqrt[4]{3}\left(t+c_{1}\right), k\right]
$$

that is

$$
\frac{\sqrt{3}-1-\sqrt[3]{\phi^{2}-1}}{\sqrt{3}+1+\sqrt[3]{\phi^{2}-1}}=c n\left[ \pm 2 \sqrt[4]{3}\left(t+c_{1}\right), k\right]
$$

In this situation $\phi$ may be written as

$$
\phi(t)= \pm\left[1+\left(\frac{\sqrt{3}-1-(\sqrt{3}+1) c n\left[ \pm 2 \sqrt[4]{3}\left(t+c_{1}\right), k\right]}{1+c n\left[ \pm 2 \sqrt[4]{3}\left(t+c_{1}\right), k\right]}\right)^{3}\right]^{\frac{1}{2}}
$$

Knowing $\phi=\frac{X}{\sqrt{b}}=X$, that is $x^{\alpha}=\frac{b-X^{2}}{a}$, which becomes $x^{\alpha}=1-X^{2}$, as $a=b=1$, the solution $x$ takes the definitive form

$$
x(t)=\left(\frac{(1-\sqrt{3})+(\sqrt{3}+1) c n\left[ \pm 2 \sqrt[4]{3}\left(t+c_{1}\right), k\right]}{1+c n\left[ \pm 2 \sqrt[4]{3}\left(t+c_{1}\right), k\right]}\right)^{\frac{1}{2}}
$$


Complex-valued solution: $a=-b=1$

This case corresponds to

$$
J=\frac{i}{3} \int\left(1-\phi^{2}\right)^{\frac{-2}{3}} d \phi=\frac{i}{3} \int \frac{d \phi}{\sqrt[3]{\left(1-\phi^{2}\right)^{2}}}
$$

where $i$ is the purely imaginary number. The equation (3.32) gives [17]

$$
\int_{0}^{\phi} \frac{d \xi}{\sqrt[3]{\left(1-\xi^{2}\right)^{2}}}= \pm 3 i\left(t+c_{2}\right)
$$

where $0 \prec \phi \prec \infty$, and $c_{2}$ is an arbitrary parameter.

The evaluation of the integral in (3.33) leads to

$$
\frac{3}{2 \sqrt[4]{3}} F(\psi, k)= \pm 3 i\left(t+c_{2}\right)
$$

The equation (3.34) may be rewritten in the form

$$
\cos \psi=c n\left[ \pm 2 i \sqrt[4]{3}\left(t+c_{2}\right), k\right]
$$

such that

$$
\frac{\sqrt{3}-1-\sqrt[3]{\phi^{2}-1}}{\sqrt{3}+1+\sqrt[3]{\phi^{2}-1}}=c n\left[ \pm 2 i \sqrt[4]{3}\left(t+c_{2}\right), k\right]
$$

From (3.36) one may get $\phi$ as

$$
\phi(t)= \pm\left[1+\left(\frac{(\sqrt{3}-1) \operatorname{cn}\left[ \pm 2 \sqrt[4]{3}\left(t+c_{2}\right), k^{\prime}\right]-(\sqrt{3}+1)}{1+c n\left[ \pm 2 \sqrt[4]{3}\left(t+c_{2}\right), k^{\prime}\right]}\right)^{3}\right]^{\frac{1}{2}}
$$

with $k^{\prime}=\sqrt{1-k^{2}}$.

In the present case, $\phi=\frac{X}{\sqrt{-1}}$, which is rewritten as $\phi=-i X$, that is $X=i \phi$, so that

$$
x^{\alpha}=-1-X^{2}=\phi^{2}-1
$$

from which one may secure the complex-valued solution $x(t)$ in the form

$$
x(t)=\left[\frac{(\sqrt{3}-1) \operatorname{cn}\left[ \pm 2 \sqrt[4]{3}\left(t+c_{2}\right), k^{\prime}\right]-(\sqrt{3}+1)}{1+c n\left[ \pm 2 \sqrt[4]{3}\left(t+c_{2}\right), k^{\prime}\right]}\right]^{\frac{1}{2}}
$$


3.2.2. Periodic and complex-valued solutions of (1.13). The equation (1.13) corresponds to $n=4$, which gives $\alpha=8$. Two cases may be studied.

Periodic solution: $a=b=1$

In this case, the integral $J$ is written in this form

$$
J=-\frac{1}{4} \int \frac{d \phi}{\sqrt[4]{\left(1-\phi^{2}\right)^{3}}}
$$

Using (1.7) one may get the equation ( [16], [17])

$$
J=-\frac{1}{4} \int_{0}^{\phi} \frac{d \xi}{\sqrt[4]{\left(1-\xi^{2}\right)^{3}}}= \pm\left(t+c_{3}\right)
$$

where $c_{3}$ is an arbitrary parameter. Therefore, by integration, the equation (3.41) reduces to

$$
\sqrt{2} F\left(\psi, \frac{\sqrt{2}}{2}\right)=\mp 4\left(t+c_{3}\right)
$$

where $0 \prec \phi \preceq 1$. From (3.42) one may ensure the following equation

$$
\cos \psi=c n\left[\mp 2 \sqrt{2}\left(t+c_{3}\right), \frac{\sqrt{2}}{2}\right]
$$

which is written in this form

$$
\sqrt[4]{1-\phi^{2}}=c n\left[\mp 2 \sqrt{2}\left(t+c_{3}\right), \frac{\sqrt{2}}{2}\right]
$$

From the equation (3.44), one may secure

$$
\phi^{2}=1-c n^{4}\left[2 \sqrt{2}\left(t+c_{3}\right), \frac{\sqrt{2}}{2}\right]
$$

Using the relation $\phi=\frac{X}{\sqrt{b}}$, that is $X=\phi$, the equation (3.45) is rewritten as

$$
X^{2}=1-c n^{4}\left[2 \sqrt{2}\left(t+c_{3}\right), \frac{\sqrt{2}}{2}\right]
$$

from which the relation $x^{\alpha}=\frac{b-X^{2}}{a}$, that is $x^{8}=1-X^{2}$, gives

$$
x(t)=\left[c n\left(2 \sqrt{2}\left(t+c_{3}\right), \frac{\sqrt{2}}{2}\right)\right]^{\frac{1}{2}}
$$

Complex-valued solution: $a=-b=1$

This condition leads to the equation [17] 


$$
\int_{0}^{x} \frac{\xi d \xi}{\sqrt{1+\xi^{8}}}= \pm i\left(t+c_{4}\right)
$$

where $0 \prec x \preceq 1$. The integral in (3.48) is known as hyperelliptic integral and its evaluation gives [18]

$$
\frac{1}{4} c n^{-1}\left[\frac{1-x^{4}}{1+x^{4}}, \frac{\sqrt{2}}{2}\right]= \pm i\left(t+c_{4}\right)
$$

which may be rearranged in the form

$$
\frac{1-x^{4}}{1+x^{4}}=c n\left[ \pm 4 i\left(t+c_{4}\right), \frac{\sqrt{2}}{2}\right]
$$

From (3.50) one may get

$$
x^{4}=\frac{1-c n\left[4 i\left(t+c_{4}\right), \frac{\sqrt{2}}{2}\right]}{1+c n\left[4 i\left(t+c_{4}\right), \frac{\sqrt{2}}{2}\right]}
$$

so that the general solution $x(t)$ may take the expression

$$
x(t)=\left(\frac{1-c n\left[4 i\left(t+c_{4}\right), \frac{\sqrt{2}}{2}\right]}{1+c n\left[4 i\left(t+c_{4}\right), \frac{\sqrt{2}}{2}\right]}\right)^{\frac{1}{4}}
$$

or in the definitive form

$$
x(t)=\left(\frac{c n\left[4\left(t+c_{4}\right), \frac{\sqrt{2}}{2}\right]-1}{c n\left[4\left(t+c_{4}\right), \frac{\sqrt{2}}{2}\right]+1}\right)^{\frac{1}{4}}
$$

Thus, the above shows that under the conditions that $\frac{a}{b}=1$, and $2 \preceq n \preceq 4$, the explicit general solutions of (1.11) are periodic.

In the sequel of this work, the related quadratic Lienard type equations to the equation (1.13) is examined.

\section{QUADRATIC LienARD TYPE EQUATIONS}

To determine the quadratic Lienard type equations related to (1.13), consider the change of variable

$$
\vartheta=x^{p}
$$

Thus one may obtain

$$
\frac{d x}{d t}=\frac{1}{p} \dot{\vartheta} \vartheta^{\frac{1-p}{p}}
$$


and

$$
\frac{d^{2} x}{d t^{2}}=\frac{1}{p} \ddot{\vartheta} \vartheta^{\frac{1-p}{p}}+\frac{1-p}{p^{2}} \dot{\vartheta}^{2} \vartheta^{\frac{1-2 p}{p}}
$$

Substituting (4.3) into (1.13) and taking into account (4.1), yields

$$
\ddot{\vartheta}+\frac{1-p}{p} \frac{\dot{\vartheta}^{2}}{\vartheta}+3 a p \vartheta^{\frac{p+4}{p}}+b p \vartheta^{\frac{p-4}{p}}=0
$$

The solution (3.47) ensures the general periodic solution of (4.4) in the form

$$
\vartheta(t)=\left(c n\left[2 \sqrt{2}\left(t+c_{3}\right), \frac{\sqrt{2}}{2}\right]\right)^{\frac{p}{2}}
$$

where $a=b=1$.

By application of $p=2$, the equation (4.4) reduces to

$$
\ddot{\vartheta}-\frac{1}{2} \frac{\dot{\vartheta}^{2}}{\vartheta}+6 \vartheta^{3}+\frac{2}{\vartheta}=0
$$

and its general solution becomes

$$
\vartheta(t)=c n\left[2 \sqrt{2}\left(t+c_{3}\right), \frac{\sqrt{2}}{2}\right]
$$

which shows for the first time that the Jacobi elliptic function $c n$ [18] may be solution of second order autonomous non-polynomial differential equations. Now a conclusion of this work may be addressed.

\section{Conclusion}

In this paper a general class of truly nonlinear oscillator equations is presented. The conditions of existence of periodic solutions are shown and periodic and explicit general solutions are examined. The general solutions of a well known Ermakov-Pinney type equation are also calculated. Finally it has been shown that the Jacobi elliptic function $c n$ may be solution of second-order autonomous non-polynomial differential equations.

Conflicts of Interest: The author(s) declare that there are no conflicts of interest regarding the publication of this paper. 


\section{REFERENCES}

[1] F. Güngör, P.J. Torres, Lie point symmetry analysis of a second order differential equation with singularity, (2017). http://arxiv.org/abs/1612.07080.

[2] M. D Monsia, Analysis of a purely nonlinear generalized isotonic oscillator equation, (2020). https://vixra.org/pdf/ 2010.0195v1.pdf.

[3] M. D. Monsia, On a nonlinear differential equation of Lienard type, (2020). https://vixra.org/pdf/2011.0050v3.pdf.

[4] E. Pinney, The nonlinear differential equation $y^{\prime \prime}+p(x) y+c y^{-3}=0$, Proc. Amer. Math. Soc. 1 (1950), 681.

[5] M. Euler, N. Euler and P. Euler, The Riccati and Ermakov-Pinney hierarchies, J. Nonlinear Math. Phys. 14(2) (2007), 290 $-310$.

[6] M. C. Nucci and P.G. L. Leach, Jacobi's Last multiplier and the complete symmetry group of the Ermakov-Pinney equation, J. Nonlinear Math. Phys. 12 (2) (2005), $305-320$.

[7] R. E. Michens, Truly Nonlinear Oscillators, World Scientific, Singapore, (2010).

[8] M. Gadella and L. P. Lara, On the solutions of a nonlinear pseudo-oscillator equation, Phys. Scripta, 89 (2014), 105205.

[9] V. R. Gorder, Continuous periodic of a nonlinear pseudo-oscillator equation in which the restoring force is inversely proportional to the dependent variable,Phys. Scripta, 90 (2015), 085208.

[10] E. A. Doutetien, A. R. Yehossou, P. Mallick, B. Rath and M. D. Monsia, On the general solutions of a nonlinear pseudooscillator equation and related quadratic lienard systems, PINSA. 86 (2020). https://doi.org/10.16943/ptinsa/2020/ 154987.

[11] L. Cveticanin, Oscillator with fraction order restoring force, J. Sound Vibration, 320 (2009), 1064 - 1077.

[12] Y. Wang, Unboundedness in a Duffing Equation with Polynomial Potentials, J. Differ. Equations, 160 (2) (2000), 467 479 .

[13] M. D. Monsia, The non-periodic solution of a truly nonlinear oscillator with power nonlinearity, (2020). https://vixra. org/pdf/2009.0174v1.pdf.

[14] S. C. Mancas and H. Rosu, Existence of periodic orbits in nonlinear oscillators of Emden-Fowler form, Phys. Lett. A, 380 (3) (2016), 422-428.

[15] J. Akande, D. K. K. Adjaï and M. D. Monsia, Theory of exact trigonometric periodic solutions to quadratic Lienard type equations, J. Math. Stat. 14 (1) (2018), $232-240$.

[16] I. S. Gradshteyn and I. M. Ryzhik, Table of Integrals, Series, and Products, Academic Press, ed. Elsevier, California, 2007.

[17] P.F. Byrd and M. D. Friedman, Handbook of Elliptic Integrals for Engineers and Physicists, Springer, Berlin, 1954.

[18] W. A. Schwalm, Lectures on Selected Topics in Mathematical Physics: Elliptic Functions and Elliptic Integrals, IOP Publishing, 2015. 